\title{
Torsion of a giant para-ovarian cyst. Conservative laparoscopic treatment
}

\author{
Dolores Borrás Suñer • Eduardo Cazorla Amorós • \\ Amaya Urgal Ayala $\cdot$ Sara Fortuño Salais • \\ César Díaz-García
}

Received: 21 December 2007 / Accepted: 1 April 2008 / Published online: 24 May 2008

(C) Springer-Verlag 2008

\begin{abstract}
Adnexal torsion is a condition that may result in serious consequences, including adnexa removal. Recently, de-torsion has replaced salpingo-oophorectomy as the treatment for a twisted ischaemic adnexum, and the best way to do it seems to be laparoscopic surgery. A case of adnexal torsion caused by a giant para-ovarian cyst treated, with good results, by a conservative laparoscopic approach is reported.
\end{abstract}

Keywords Adnexal torsion - Large ovarian cyst .

Laparoscopic management - Ovarian cyst

\section{Introduction}

Adnexal torsion (AT) is the fifth most common surgical gynaecological emergency and, therefore, requires prompt diagnosis and treatment. Mostly, it occurs in the childbearing age group, with a frequency of $2.7 \%$ of all gynaecological emergencies, but it is not uncommon in premenarchal girls $(15 \%)$ or postmenopausal women $(15 \%)$ [1]. This entity consists of total or partial rotation of the adnexum around its vascular axis that induces a venous and lymphatic blockage leading to gangrene and haemorrhagic necrosis. It usually presents as a sudden, continuous, nonspecific pain in the lower abdomen. When AT is suspected, a prompt laparoscopic approach is indicated to confirm the diagnosis and to avoid irreversible ovarian damage [2]. Ultrasound findings and Doppler flow study, in addition to

D. Borrás Suñer $(\bowtie) \cdot$ E. Cazorla Amorós $\cdot$ A. Urgal Ayala $\cdot$

S. Fortuño Salais · C. Díaz-García

Unit of Gynecological Endoscopy, Gynecology Department,

La Fe University Hospital,

Av. Campanar 21,

46009 Valencia, Spain

e-mail: doloresborras@hotmail.com the clinical symptoms, may help to achieve diagnosis [3]. Definitive diagnosis would be with laparoscopy or laparotomy examination. The aetiology is unknown. In most cases, an ovarian tumour is present. Torsion of a normal sized ovary is extremely rare. Large and heavy ovarian cysts, such as benign cystic teratoma or polycystic ovaries, seem to be prone to torsion. Comerci et al [4] reported an incidence of $3.5 \%$ torsions in a series of 517 patients with benign cystic teratomas. Cysts smaller than $5 \mathrm{~cm}$ are infrequently a cause of torsion. After pelvic inflammatory disease and pelvic endometriosis, torsion is rare, owing to associated pelvic adhesions, even if an endometrioma is present. Until recently, the standard management of the twisted ischaemic adnexum was salpingo-oophorectomy. Recently, it has been suggested that de-torsion is an alternative mode of treatment $[5,6]$. The advantages of ovarian preservation are clear, because AT occurs most often in adolescent girls and in women of child-bearing age. Although the size of the cyst is often the limiting factor for laparoscopic management, in selected cases it may be a safe option.

\section{Case report}

A 25-year-old woman, gravida 3, para 3, with regular menstrual cycles, intrauterine device as a contraceptive method, and with previous history of renal colic, was admitted to the hospital after the onset of right renal pain with nausea and vomiting. There was tenderness on deep abdominal palpation, with evident abdominal guarding. An ultrasound scan revealed a large cystic mass of dense content that appeared to depend on the right adnexum and was on the uterus. The mass measured $117 \mathrm{~mm} \times 89 \mathrm{~mm}$. Colour and spectral Doppler findings were absence of vascularisation both peripherally and inside the mass, 


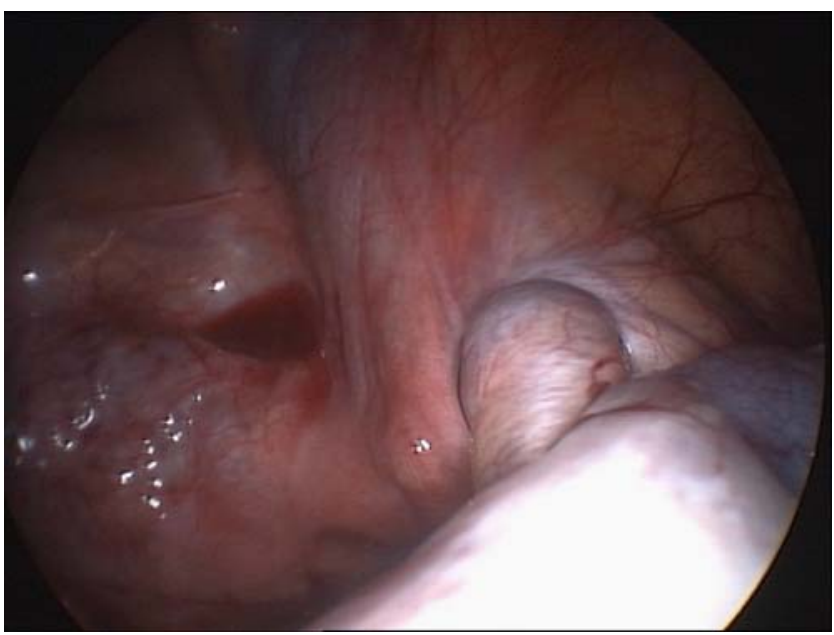

Fig. 1 Intraoperative findings of right adnexal torsion

according with signs of hypoperfusion. The blood investigations revealed moderate anaemia and leukocytosis with left deviation, serum tumour marker values (CA125, CA19.9) within the normal ranges, and negative findings for serum beta human chorionic gonadotropin (beta-hCG).

An emergency laparoscopy was performed, using standard equipment. The examination of the pelvis showed a slight increase in the size of the right ovary, torsion of the tube, and a para-ovarian cyst of $150 \mathrm{~mm}$, with 3-4 turns around its axis (Fig. 1). The left ovary had a trophic aspect. After puncture (Fig. 2) and evacuation of yellowish-white homogeneous fluid, de-torsion (Fig. 3) and cystectomy (Fig. 4) was achieved, obtaining the integrity of tube and ovary with any damage during the surgical procedure. The postoperative course was unremarkable, and the patient was discharged home on day 2; histological examination was reported as indicative of a papillary serous cyst. One month after treatment the patient was asymptomatic, and ultrasound scan revealed normal adnexa.

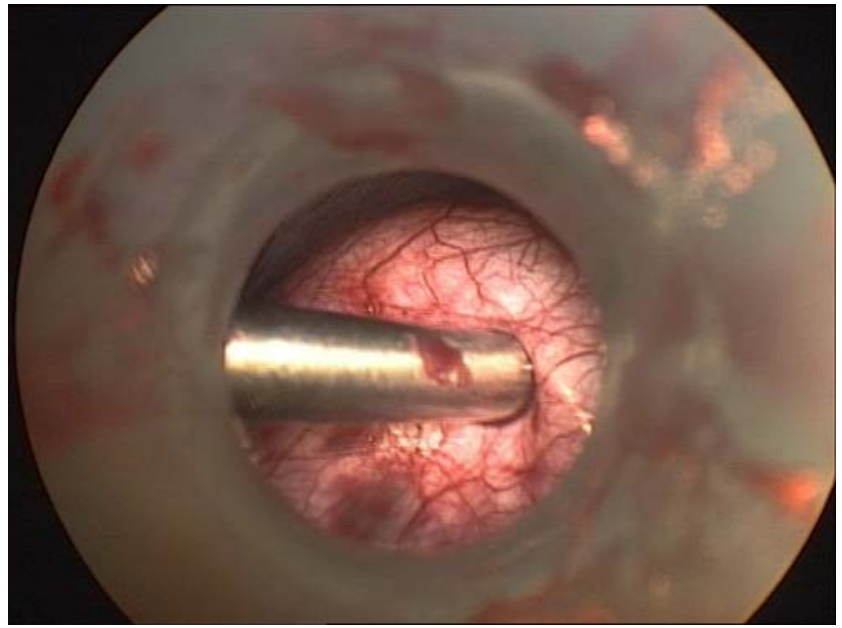

Fig. 2 First step: puncture and evacuation of cystic content

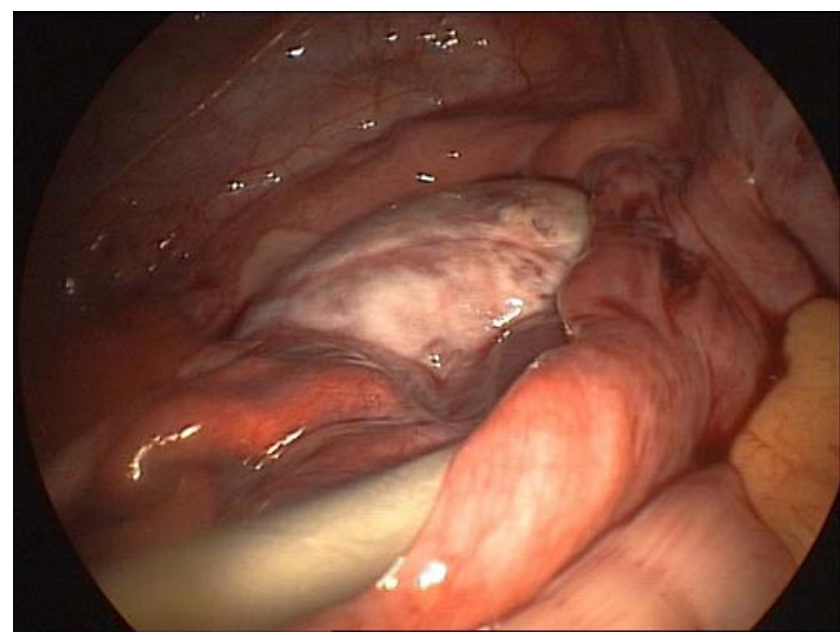

Fig. 3 Second step: de-torsion

\section{Discussion}

AT usually occurs during reproductive age, which makes this gynaecological emergency more important. If diagnosis is early, AT should be managed conservatively. On the other hand, delays in diagnosis and surgical treatment are common in AT because of non-specific clinical findings. Nausea and vomiting are quite common, occurring in $70 \%$ of patients. Pyrexia may also be present. There are no specific laboratory findings in AT, although the white blood count may be elevated, with no correlation between leukocyte count and tissue necrosis. Definitive diagnosis depends on direct visualisation by laparotomy or laparoscopy.

Torsion of the adnexa should be suspected in any patient presenting with abdominal pain and a recognised risk factor for ovarian torsion. These risk factors include conditions such as previous abdominal surgery, past history of ovarian torsion, previous or current ovarian cyst, ovarian hyperstimulation, polycystic ovaries and tubal ligation [7].

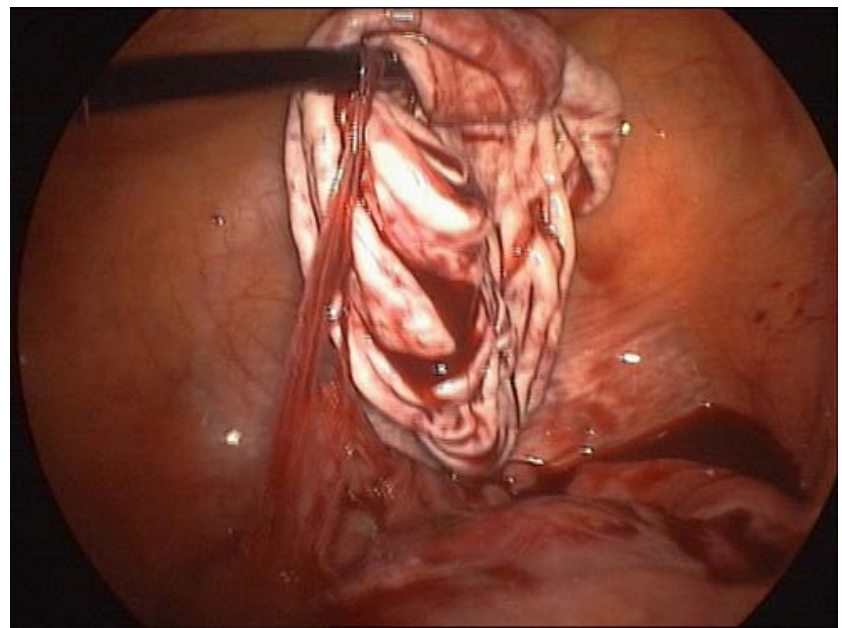

Fig. 4 Third step: cystectomy 
The clinical features of torsion can be non-specific, and the differential diagnosis is wide and should include the consideration of other surgical emergencies, such as ectopic pregnancy, ovarian cyst, pelvic mass, gynaecological infection, renal colic, appendicitis and gastrointestinal complaints. In our case, we could think of renal colic because of the patient's medical history.

Complete or partial torsion of the ovarian vascular pedicle initially compromises lymphatic and venous drainage, with eventual loss of arterial perfusion. The ultrasound scan findings of AT vary, depending on the degree of vascular compromise and the presence of an adnexal mass [1]. The most frequent significant sign of ovarian torsion is the presence of multiple cortical follicles in an enlarged ovary [8]. Colour Doppler suggests AT when there is diminished or absent vascularisation, and spectral Doppler gives information about the type of flow (arterial or venous). Venous flow is the first that disappears, but arterial flow can be present for a longer time [9]. In this patient both colour and spectral Doppler showed findings of the final step of complete AT, because no venous or arterial flow was demonstrated. In the largest study published in the literature on the effectiveness of Doppler examination in predicting ovarian torsion, Ben-Ami et al. [9] reported a positive predictive value of $94 \%$ if venous flow is absent.

Up until the 1990s, AT had been managed by adnexectomy because of the belief that de-torsion would increase the risk of pulmonary embolism from the twisted pedicle, and the necrotic ovary could predispose the patient to disseminated intravascular coagulation or pulmonary collapse with the release of vasoactive substances from ischaemic tissues [10]. However, some authors have demonstrated that de-torsion of the twisted adnexa preserves ovarian function and does not increase the risk of pulmonary embolism, in comparison with excision without untwisting [11, 12]. The patient we have report on did not have any complication. Several studies have shown that estimation of the degree of necrosis during surgery is inaccurate [13]. Colour, size and oedema of the twisted ischaemic adnexa usually do not reflect the real damage to the ovarian tissue, and, probably, the colour is due to venous stasis, because venous flow is the first to be compromised. On the other hand, the ovary has double arterial irrigation, from the aorta and the uterine arteries, that allows long periods of torsion without ischaemia; thus, de-torsion is probably the only technical treatment. The twisted ischaemic adnexum appears oedematous and friable, and it is liable to be traumatised. De-torsion should, therefore, be performed with care, and preferably with blunt instruments if laparoscopy is used. Ovarian function is preserved in $88 \%$ to $100 \%$ of cases [14]. There is no need to extend the duration of operation to observe ovarian reperfusion. Recurrence of ovarian torsion is rare.
De-torsion can be performed by laparoscopy or laparotomy. Laparoscopy is associated with a shorter length of hospitalisation, with a mean of $2.1 \pm 1.2$ days against $7.4 \pm$ 1.5 for laparotomy [12], and a greater degree of patient comfort. Therefore, laparoscopy is the preferred approach whenever experienced laparoscopists are available. In our case, she was at hospital for 2 days.

Some authors have studied ovarian function with ultrasound, evaluating blood flow with Doppler colour, size of the ovary, and follicular development, and showing follicular development and normal sized ovary in $93 \%$ of cases [12]. Unfortunately, there is no way to predict which ovaries that have been severely injured will recover. However, leaving the necrotic and non-viable ovaries in situ did not cause further injury to the patient.

In conclusion, torsion of the adnexa is considered a surgical emergency to preserve reproductive function. The gold standard in its management is laparoscopy, and this justifies the importance of skill in using laparoscopy in emergency surgery as well as the approach with this technique in giant cysts, in selected cases.

\section{References}

1. Bassil S, Steinhart U, Donnez J (1999) Successful laparoscopic management of adnexal torsion. Hum Reprod 14:855-857

2. White M, Stella J (2005) Ovarian torsion: 10-years perspective. Emerg Med Australas 17:231-237

3. Cil AP, Akgul MA, Tulunay G, Atayar YY (2006) Recovery of ovarian function after detorsion: Doppler findings. Acta Radiol 47:618-620

4. Comerci JT Jr, Licciardi F, Bergh PA, Gregori C, Breen JL (1994) Mature cystic teratoma: a clinico-pathologic evaluation of 517 cases and review of the literature. Obstet Gynecol 84:22-28

5. Mage G, Canis M, Manhes H, Pouly JL, Bruhat MA (1989) Laparoscopic management of adnexal torsion. A review of 35 cases. J Reprod Med 34:520-524

6. Wagaman R, Williams RS (1990) Conservative therapy for adnexal torsion. A case report. J Reprod Med 35:833-834

7. Houry D, Abbott JT (2001) Ovarian torsion: a fifteen-year review. Ann Emerg Med 38:156-159

8. Lambert MJ, Villa M (2004) Gynecologic ultrasound in emergency medicine. Emerg Med Clin North Am 22:683-696

9. Ben-Ami M, Perlitz Y, Haddad S (2002) The effectiveness of spectral and color Doppler in predicting ovarian torsion. A prospective study. Eur J Obstet Gynecol Reprod Biol 104:64-66

10. Oelsner G, Bider D, Goldenberg M (1993) Long term follow up of the twisted ischemic adnexa managed by torsion. Fertil Steril 60:976-979

11. McGovern PG, Noah R, Koenigsberg R, Little AB (1999) Adnexal torsion and pulmonary embolism: case report and review of the literature. Obstet Gynecol Surv 54:601-608

12. Oelsner G, Cohen SB, Soriano D, Admon D, Mashiach S, Carp H (2003) Minimal surgery for the twisted ischemic adnexa can preserve ovarian function. Human Reprod 18:2599-2602

13. Mazouni C, Bretelle F, Menard JP, Blanc B, Gamerre M (2005) Diagnosis of adnexal torsion and predictive factors of adnexal necrosis. Gynecol Obstet Fertil 33:102-106

14. Oelsner G, Shashar D (2006) Adnexal torsion. Clin Obstet Gynecol 49:459-463 